\title{
PNPLA3 rs738409 Polymorphism Associated with Hepatic Steatosis and Advanced Fibrosis in Patients with Chronic Hepatitis C Virus: A Meta-Analysis
}

\author{
Jia-Hao Fan*, Ming-Que Xiang ${ }^{\dagger}$, Qing-Ling Li ${ }^{\ddagger}$, Hong-Tao Shi*, and Jin-Jun Guo* \\ ${ }^{*}$ Department of Gastroenterology and Hepatology, The Second Affiliated Hospital of Chongqing Medical University, ${ }^{\dagger}$ Department of Infectious \\ Diseases, The Ninth People’s Hospital of Chongqing, and ${ }^{\ddagger}$ Institute of Life Sciences, Chongqing Medical University, Chongqing; China
}

\begin{abstract}
Background/Aims: The recognition of a correlation between patatin-like phospholipase domain containing-protein 3 (PNPLA3) rs738409 (C>G) and the severity of liver steatosis or fibrosis in chronic hepatitis $\mathrm{C}(\mathrm{CHC})$ has not reached a consensus. This meta-analysis sought to investigate with accuracy the association between the PNPLA3 rs 738409 (C>G) polymorphism and liver steatosis and advanced fibrosis in CHC patients. Methods: We performed a comprehensive literature search from the PubMed, Embase, Web of Science, and Google Scholar databases up to December 31, 2014. Crude odds ratios (ORs) with 95\% confidence intervals (Cls) were calculated. Statistical analyses were performed using Stata 12.0 software. Results: The meta-analysis revealed the severity of liver fibrosis was significantly higher in $\mathrm{CHC}$ patients with PNPLA3 rs738409 GG in Caucasians (versus CC+CG: OR, 2.29; 95\% Cl, 1.57 to 3.35; $p<0.05$ ) but not Asian populations. In Caucasians, liver steatosis was also more severe in $\mathrm{CHC}$ patients with rs $738409 \mathrm{GG}$ (versus $\mathrm{CC}+\mathrm{CG}$; OR, 4.33; 95\% Cl, 2.59 to 7.22; $p<0.05$ ). The sensitivity analysis indicated the results of this meta-analysis were stable and no publication bias was detected. Conclusions: PNPLA3 rs738409 (C>G) was associated with the risk of both advanced liver fibrosis and steatosis in patients with $\mathrm{CHC}$, especially among Caucasian populations. (Gut Liver 2016;10:456-463)
\end{abstract}

Key Words: Patatin-like phospholipase domain containing protein 3 rs738409; Advanced liver fibrosis; Fatty liver; Hepatitis C, chronic; Meta-analysis

\section{INTRODUCTION}

Nearly three percent of the worldwide population has been infected with the hepatitis C virus (HCV) and about 80\% of these cases are chronic. ${ }^{1,2}$ Chronic hepatitis $\mathrm{C}$ (CHC) infection has become one of the leading causes of liver-related mortality. Yet, the clinical progression of CHC-related liver diseases is highly variable. ${ }^{3}$ Epidemiological studies have shown that the severity of liver disease in $\mathrm{CHC}$ is associated with older age, male gender, higher body mass index (BMI), insulin resistance, chronic alcohol consumption ( $>30$ to $50 \mathrm{~g} /$ day), viral factors (especially HCV genotype 3), or coinfection with human immunodeficiency virus. ${ }^{4-8}$ However, even when combined these factors have an overall prediction rate of less than 30\%. ${ }^{9}$ Investigative research has turned to the influence of genetics.

Several potential genetic risk factors have been identified as having a key role in the severity of CHC-associated liver disease. The best documented is the polymorphism rs738409 (C>G) of patatin-like phospholipase domain-containing protein 3 (PNPLA3), encoding the 148 isoleucine to methionine protein variant (p.I148M). Nevertheless, recognition of a correlation between PNPLA3 rs73409 (C>G) and the severity of liver steatosis or fibrosis in $\mathrm{CHC}$ has not reached a consensus. Several studies have indicated that $\mathrm{CHC}$ patients who carry the GG genotype of PNPLA3 rs738409 (C>G) are at high risk of advanced liver fibrosis, ${ }^{10-12}$ but a similar result is not shown in others. ${ }^{13}$ Moreover, among the studies that found a significant association the strength of the correlation varied, ranging from odds ratios (ORs) of $1.88^{11}$ to $7.53^{10}$ for advanced liver fibrosis, and $3.12^{11}$ to $8.78^{14}$ for liver steatosis.

By increasing the sample size, the objective of this metaanalysis was to explore more accurately the association between

\footnotetext{
Correspondence to: Jin-Jun Guo

Department of Gastroenterology and Hepatology, The Second Affiliated Hospital of Chongqing Medical University, 76 Linjiang Road, Yuzhong District, Chongqing 400010, China

Tel: +86-02363693326, Fax: +86-02368486780, E-mail: guojinjun1972@163.com

Received on June 11, 2015. Revised on June 25, 2015. Accepted on June 25, 2015. Published online September 30, 2015

pISSN 1976-2283 eISSN 2005-1212 http://dx.doi.org/10.5009/gnl15261

Jia-Hao Fan and Ming-Que Xiang contributed equally to this work as first authors.

@ This is an Open Access article distributed under the terms of the Creative Commons Attribution Non-Commercial License (http://creativecommons.org/licenses/by-nc/4.0) which permits unrestricted non-commercial use, distribution, and reproduction in any medium, provided the original work is properly cited.
} 
PNPLA3 rs738409 (C>G) and liver steatosis and advanced fibrosis in patients with $\mathrm{CHC}$ infection.

\section{MATERIALS AND METHODS}

\section{Literature search and study selection}

Relevant studies published before December 31, 2014 were found by searching the PubMed, Embase, and Web of Science databases, and the Google Scholar website for the query "(patatin-like phospholipase domain containing 3 or PNPLA3) and (rs738409 or I148M) and (fibrosis or steatosis) and (chronic hepatitis C or CHC or hepatitis C virus or HCV)." There were no language restrictions. Other potential sources were searched for in the references of qualified articles. Approval from the ethics committee was not required because we only used data that had been previously published. All study selection and data extraction was performed independently by two authors. Disagreements were resolved by discussion with a third author. A flow chart of the study selection process is shown in Fig. 1.

\section{Study inclusion and exclusion criteria}

The diagnosis of HCV infection in all patients of the selected studies was confirmed through serological examinations. Fibrosis and steatosis were confirmed by liver biopsy. Advanced

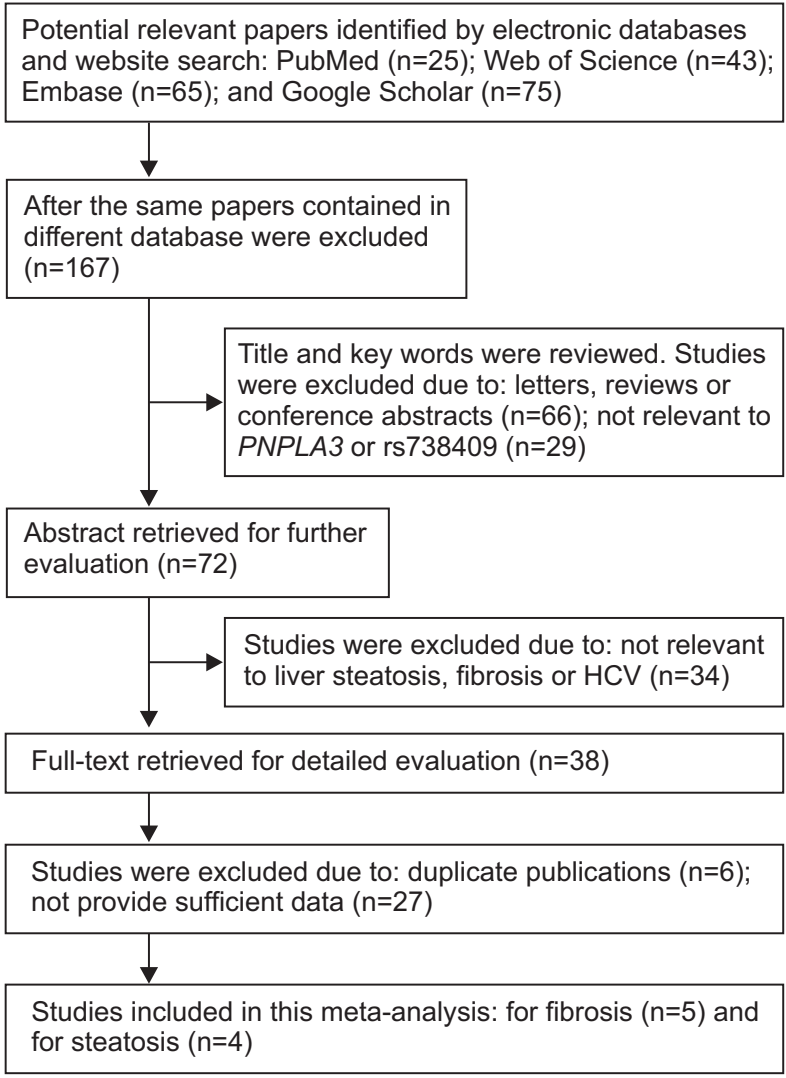

Fig. 1. Schematic of the study selection process. $\mathrm{HCV}$, hepatitis $\mathrm{C}$ virus. fibrosis was defined as stage F3 (numerous septa without cirrhosis) or F4 (cirrhosis) according to the METAVIR score. The extent of steatosis was analyzed by calculating the percentage of lipid-containing hepatocytes in the total number of hepatocytes.

The selected studies conformed to the following criteria: (1) the studies focused on the polymorphism rs738409 (C>G) of PNPLA3 and fibrosis or steatosis in patients with CHC infection; (2) with sufficient reported data (stage of fibrosis or steatosis, and stratified outcomes according to PNPLA3 genotype); (3) fit the diagnostic criteria for HCV infection, liver fibrosis and steatosis; and (4) the study was conducted in humans. If the same patient population was included in more than one study, the highest quality evaluation or the largest sample size was included. Letters, reviews or abstracts, and proceedings from clinical conferences were excluded.

\section{Data extraction}

The following data of each study was extracted independently by two authors: the first author, year of publication, ethnicity of subjects, age, BMI, gender ratio, HCV genotype, treatments, accompanying hepatitis B virus (HBV) or human immunodeficiency virus (HIV) infection, number of subjects, genotype frequencies of single nucleotide polymorphisms, and Hardy-Weinberg equilibrium (HWE). Results that were not reported directly were calculated from the data by two authors independently. Disagreements were resolved by discussion with a third author.

\section{Quality assessment of the included studies}

Two authors independently assessed the quality of the included studies based on the Newcastle-Ottawa Scale (http://www. ohri.ca/programs/clinical_epidemiology/oxford.asp). ${ }^{15}$ The semiquantitative star system, containing (selection, comparability, and exposure assessment) was applied. Disagreements were resolved by discussion with a third author.

\section{Statistical analyses}

The HWE of each study was tested using the chi-square test. Based on previous studies, ${ }^{16}$ the ORs, and 95\% confidence intervals (CIs) were calculated under the recessive genetic model (i.e., GG compared to $\mathrm{CC}+\mathrm{CG}$ ). The $\mathrm{Z}$ test and $\mathrm{p}<0.05$ were used to examine the statistical significance of the pooled ORs.

Cochran's Q-test and the $\mathrm{I}^{2}$ test were used to evaluate the heterogeneity between the studies. ${ }^{17,18}$ If $\mathrm{p} \geq 0.1$ of the Q-test, or $\mathrm{I}^{2} \leq 50 \%$, we considered that no significant heterogeneity existed, so the fixed effects model (Mantel-Haenszel method) was used in this meta-analysis. If not, the random effects model (DerSimonian and Laird method) would be considered. A subgroup analysis by ethnicity or genotype of HCV was performed to find sources of heterogeneity.

Sensitivity was determined by repeating the analysis and evaluating the stability of each result, after omitting in turn the 
Table 1. Characteristics of the Cohorts of the Included Studies*

\begin{tabular}{|c|c|c|c|c|c|c|c|c|c|c|c|}
\hline \multirow{3}{*}{ Author (year) } & \multirow{3}{*}{ Ethnicity } & \multirow{3}{*}{$\begin{array}{c}\text { HCV } \\
\text { genotype }\end{array}$} & \multicolumn{4}{|c|}{ Fibrosis } & \multicolumn{4}{|c|}{ Steatosis } & \multirow{3}{*}{$\begin{array}{c}\text { HWE } \\
\text { p-value }\end{array}$} \\
\hline & & & \multicolumn{2}{|c|}{ Mild or none } & \multicolumn{2}{|c|}{ Advanced } & \multicolumn{2}{|r|}{$<5 \%$} & \multicolumn{2}{|r|}{$\geq 5 \%$} & \\
\hline & & & $\mathrm{n}$ & $\mathrm{CC}+\mathrm{CG} / \mathrm{GG}$ & $\mathrm{n}$ & $\mathrm{CC}+\mathrm{CG} / \mathrm{GG}$ & $\mathrm{n}$ & $\mathrm{CC}+\mathrm{CG} / \mathrm{GG}$ & $\mathrm{n}$ & $\mathrm{CC}+\mathrm{CG} / \mathrm{GG}$ & \\
\hline Stattermayer et al. (2012) & Caucasian & 1,4 & 159 & $155 / 4$ & 43 & $36 / 7$ & 87 & $85 / 2$ & 115 & $106 / 9$ & 0.56 \\
\hline Valenti et al. (2012) ${ }^{11}$ & Caucasian & $1,2,3,4$ & 423 & $395 / 28$ & 179 & $158 / 21$ & 196 & $189 / 7$ & 406 & $364 / 42$ & 0.44 \\
\hline Stattermayer et al. (2013) & Caucasian & 1,4 & 322 & $308 / 14$ & 156 & $138 / 18$ & 147 & $145 / 2$ & 213 & $190 / 23$ & 0.69 \\
\hline Trepo et al. $(2011)^{14}$ & Caucasian & $1,2,3,4$ & 321 & $306 / 15$ & 216 & $193 / 23$ & 264 & $257 / 7$ & 273 & $242 / 31$ & 0.92 \\
\hline Miyashita et al. (2012) ${ }^{13}$ & Asian & 1,2 & 161 & $129 / 32$ & 57 & $43 / 14$ & - & - & - & - & 0.59 \\
\hline
\end{tabular}

$\mathrm{HCV}$, hepatitis C virus; HWE, Hardy-Weinberg equilibrium.

${ }^{*}$ All studies were in HWE.

data of each study. To assess the potential publication bias, we used Begger's funnel plot and Egger's linear regression test. ${ }^{19}$ Stata software for Windows version 12.0 (Stata, College Station, TX, USA) was used for all the statistical analyses.

\section{RESULTS}

\section{Characteristics of included studies}

Five studies were relevant to advanced fibrosis of the liver in CHC and comprised 2,037 patients. There were no statistically significant differences among them with regard to age, BMI, or gender ratio (specific data are not listed), including 651 patients with advanced fibrosis (METAVIR stages F3-F4). In four of these the subjects were Caucasian, and in one the subjects were Asian (Table 1). The four studies with Caucasian subjects also concerned steatosis of the liver with, in total, 694 subjects in whom the extent of steatosis was more than 5\%. All of the patients had performed liver biopsies before received the treatment of pegylated interferon and ribavirin, and were without HBV or HIV infection.

Based on the results of the line probe assay VERSANT HCV Genotype 2.0 (Siemens, Tarrytown, NY, USA), two studies ${ }^{10,12}$ included patients infected with the HCV genotypes 1 or 4 ; in one study, ${ }^{13}$ patients were infected with HCV genotypes 1 or 2 ; and in the remaining two studies, ${ }^{11,14}$ patients were infected with HCV genotypes 1, 2, 3, or 4. The genotyping of PNPLA3 (rs738409) was performed using the TaqMan Assay (Applied Biosystems, Foster City, CA, USA). After calculation, all allele frequencies were in $\mathrm{HWE}(\mathrm{p}>0.05)$.

\section{Association between PNPLA3 rs738409 (C>G) and advanced fibrosis}

With regard to the association between PNPLA3 rs738409 $(\mathrm{C}>\mathrm{G})$ and advanced fibrosis in CHC patients, in this meta-analysis interstudy heterogeneity was found, but was not significant $\left(\mathrm{I}^{2}=37.7 \%, \mathrm{p}=0.170\right)$; therefore the fixed effects model (MantelHaenszel method) was used to calculate the pooled OR and 95\% CI. The genotype GG of PNPLA3 rs738409 (C>G) was associated with higher risk of advanced liver fibrosis in patients with $\mathrm{CHC}$ (GG versus CC+CG: OR, 2.19; 95\% CI, 1.59 to 3.02; p<0.05) (Fig. 2A).

The subgroup analysis by ethnicity showed that in the Caucasian populations, CHC patients with genotype GG had a higher risk of advanced fibrosis of the liver compared with those with genotype CC+CG (OR, 2.51; 95\% CI, 1.75 to 3.60; $\mathrm{p}<0.05$ ). However, this association was not observed in the Asian population (OR, 1.31; 95\% CI, 0.65 to 2.67 ; $\mathrm{p}=0.457$ ).

We further performed a subgroup analysis based on HCV genotype in Caucasians. Subgroup A included two studies ${ }^{10,12}$ in which the CHC patients were infected with HCV-1 or HCV-4 only. Subgroup B included the other two studies ${ }^{11,14}$ comprising $\mathrm{CHC}$ patients infected with not only HCV-1 or HCV-4 but also $\mathrm{HCV}-2$ or HCV-3. Although the two subgroups differed, the difference was not significant (subgroup A: OR, 3.57; 95\% CI, 1.90 to 6.69; subgroup B: OR, 2.11; 95\% CI, 1.35 to 3.29; p=0.17) (Fig. 2B).

\section{Association between PNPLA3 rs738409 (C>G) and steatosis}

With regard to the association between PNPLA3 rs738409 (C>G) polymorphism and steatosis in CHC patients, in this metaanalysis interstudy heterogeneity was not observed $\left(\mathrm{I}^{2}=0.0 \%\right.$, $\mathrm{p}=0.658$ ), and therefore the fixed effects model (Mantel-Haenszel method) was used to calculate the pooled OR and 95\% CI. The genotype GG of PNPLA3 rs738409 (C>G) was associated with the risk of steatosis of the liver in patients with $\mathrm{CHC}$ (GG versus CC+CG: OR, 4.33; 95\% CI, 2.59 to 7.22; p<0.05) (Fig. 3).

We also performed a subgroup analysis stratified by HCV genotype, whereby in subgroup $\mathrm{A}$, the $\mathrm{CHC}$ patients were infected with HCV-1 or HCV-4 only, ${ }^{10,12}$ and in subgroup B, the CHC patients were infected with HCV-1, 2, 3, or $4 .{ }^{11,14}$ Although the two subgroups differed, the difference was not significant (subgroup A: OR, 6.20; 95\% CI, 2.16 to 17.79; subgroup B: OR, 3.79 ; 95\% CI, 2.11 to $6.68 ; \mathrm{p}=0.49$ ).

\section{Sensitivity analysis}

For the sensitivity analysis we used the random effects model 


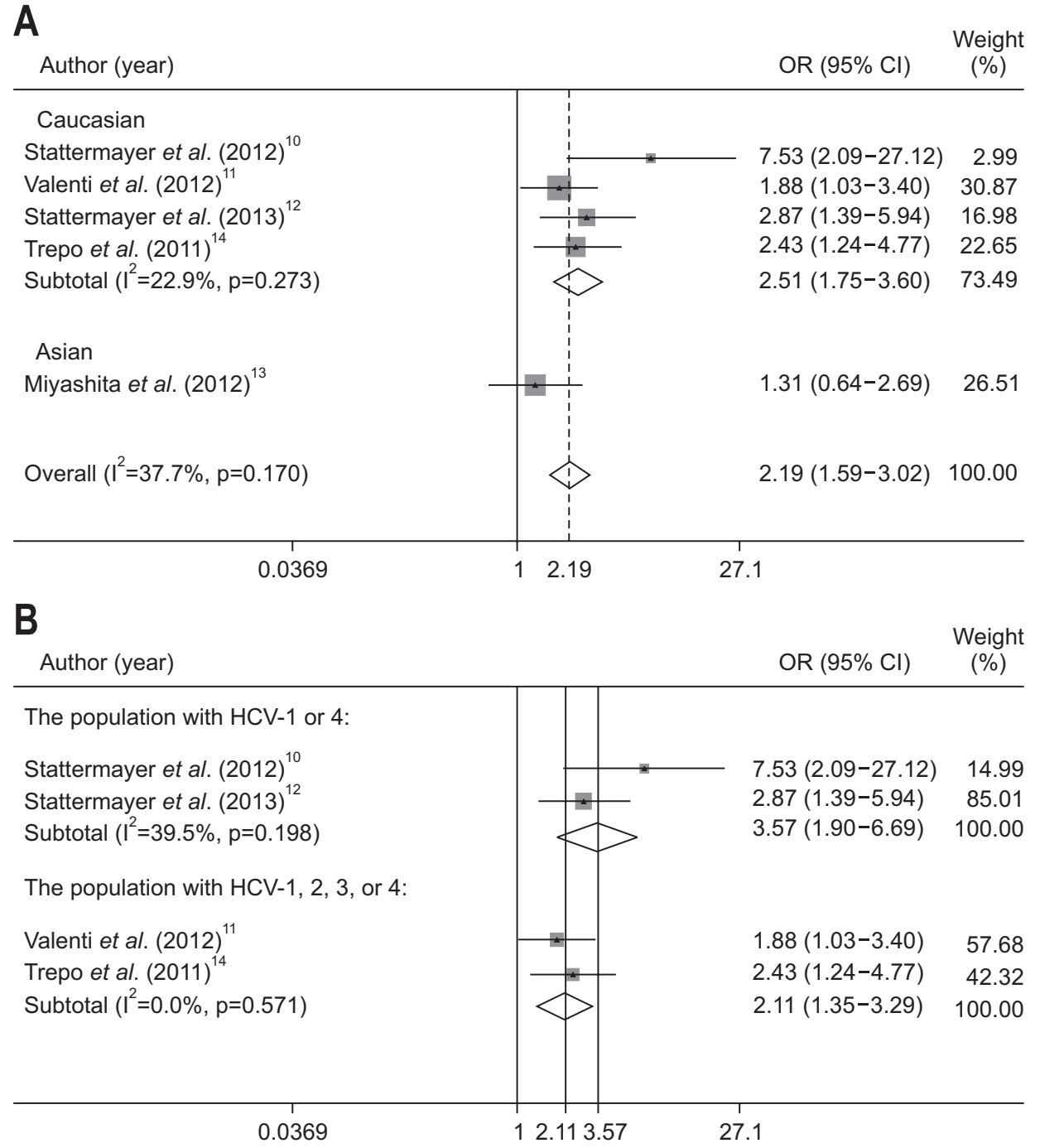

Asian

Miyashita et al. (2012)

Overall $\left(I^{2}=37.7 \%, p=0.170\right)$
Weight erimates for odds ratios (ORs, effect), the corresponding 95\% confidence interval (CI) limits (lower and upper), percent weight, and overall or subtotal ( $\mathrm{I}^{2}$ and $\mathrm{p}$-value) were calculated by fixed effects metaanalysis for advanced fibrosis. The first author of the study and the year of publication are shown. (A) The forest plots of all five studies were relevant to advanced fibrosis and the subgroup analysis by ethnicity. (B) The forest plots of all five studies were relevant to advanced fibrosis and the subgroup analysis based on hepatitis $\mathrm{C}$ virus (HCV) genotype.

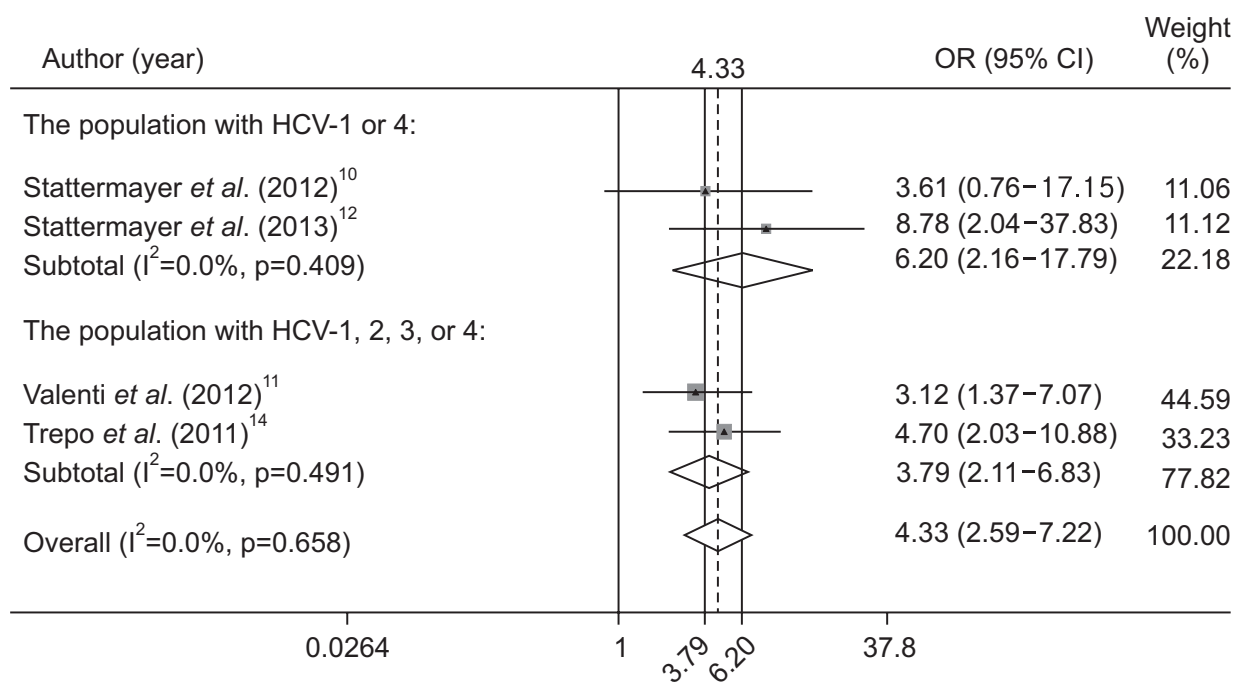

Fig. 3. Liver steatosis. Summary estimates for odds ratios (ORs, effect), the corresponding 95\% confidence interval (CI) limits (lower and upper), percent weight, and overall or subtotal ( $\mathrm{I}^{2}$ and $\mathrm{p}$-value) were calculated by fixed effects meta-analysis for liver steatosis. The forest plots of all five studies were relevant to liver steatosis and the subgroup analysis based on hepatitis $\mathrm{C}$ virus (HCV) genotype. 
Meta-analysis random-effects estimates (exponential form)

study ommited

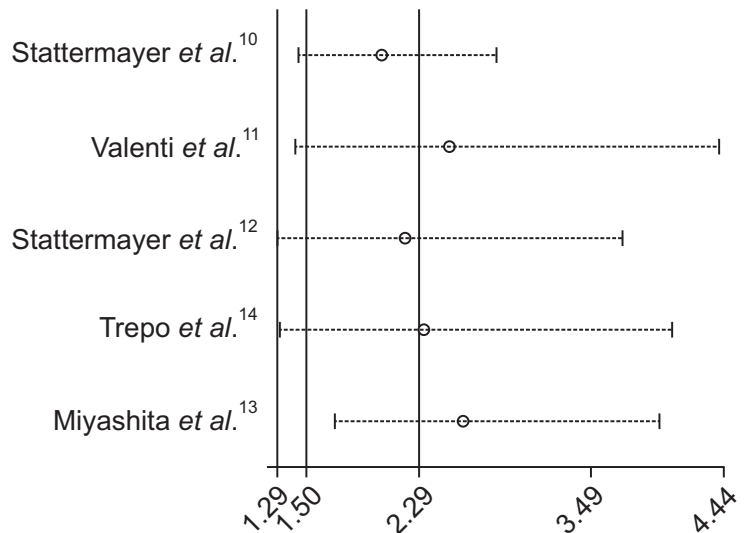

B

Meta-analysis random-effects estimates (exponential form) study ommited

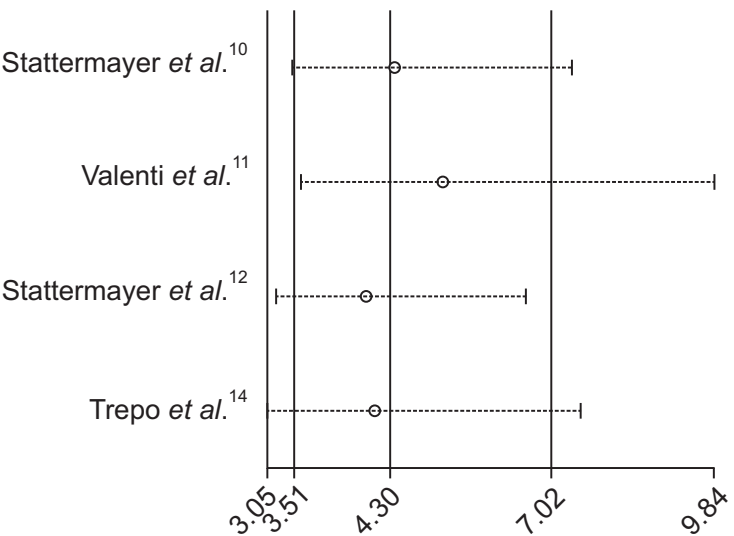

Fig. 4. Sensitivity analysis of the summary odds ratio. The results were computed by omitting each study in turn. Meta-analysis random effects estimates (exponential form) were used. The two ends of the dotted lines represent the 95\% confidence interval. (A) Association between patatin-like phospholipase domain-containing protein 3 (PNPLA3) rs738409 single nucleotide polymorphism (SNP) and advanced fibrosis. (B) Association between PNPLA3 rs738409 SNP and liver steatosis.

A Begg's funnel plot with pseudo 95\% confidence limits

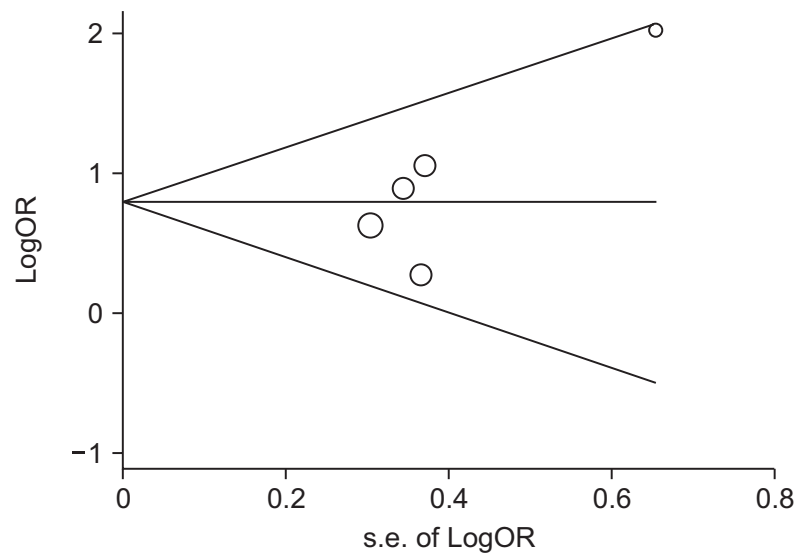

B Begg's funnel plot with pseudo 95\% confidence limits

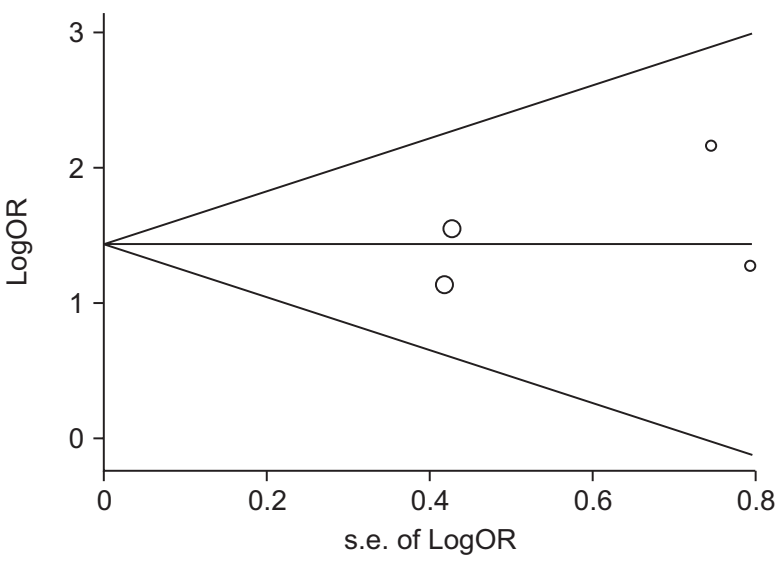

Fig. 5. Begger's funnel plot. Each point represents a separate study for the indicated association. Horizontal line means magnitude of the effect. (A) Association between patatin-like phospholipase domain-containing protein 3 (PNPLA3) rs738409 single nucleotide polymorphisms (SNP) and advanced fibrosis. (B) Association between PNPLA3 rs738409 SNP and liver steatosis.

s.e., standard error; LogOR, natural logarithm of odds ratio.

to recalculate the pooled ORs (95\% CIs), and there were no significant changes in results. Furthermore, when any single study was deleted, the corresponding pooled ORs were not substantially altered (Fig. 4), suggesting that the results of this metaanalysis were stable.

\section{Publication bias}

Begger's funnel plots (Fig. 5) and Egger's linear regression test were performed to assess the publication bias of all included studies. The results indicated no evidence of publication bias (advanced fibrosis, $\mathrm{p}=0.144$; steatosis, $\mathrm{p}=0.504$ ).

\section{Quality assessment}

Based on the Newcastle-Ottawa Scale, the quality evaluations of the included studies showed that all five of the included studies earned more than five stars, and therefore could be considered medium-high quality reports (Table 2). Disagreements were also resolved by discussion with a third author.

\section{DISCUSSION}

This meta-analysis investigated whether the PNPLA3 polymorphism rs738409 $(\mathrm{C}>\mathrm{G})$ is related to liver steatosis or advanced fibrosis in CHC patients. We conducted a search for relevant papers published up to June 1, 2014 in the PubMed, Embase, Web of Science, and Google Scholar databases, as well as the papers cited in these articles. Five studies satisfied the inclusion criteria, in which the cohorts of four were Cau- 
Table 2. Quality of the Included Studies

\begin{tabular}{|c|c|c|c|}
\hline Author (year) & Selection & Comparability & Exposure/outcome \\
\hline Stattermayer et al. (2012) & $\star \star \star$ & $\star \star$ & $\star \star \star$ \\
\hline Valenti et al. (2012) ${ }^{11}$ & $\star \star$ & $\star \star$ & $\star \star \star$ \\
\hline Stattermayer et al. (2013) ${ }^{12}$ & $\star \star \star$ & $\star \star$ & $\star \star \star$ \\
\hline Trepo et al. $(2011)^{14}$ & $\star \star$ & $\star \star$ & $\star \star \star$ \\
\hline Miyashita et al. (2012) ${ }^{13}$ & $\star \star \star \star$ & $\star \star$ & $\star \star$ \\
\hline
\end{tabular}

casians, and in one the subjects were Asian. It was determined that PNPLA3 rs738409 $(\mathrm{C}>\mathrm{G})$ was associated with risk of both advanced liver fibrosis and steatosis in patients with CHC. The association was especially strong among Caucasians.

PNPLA3 rs738409 (C>G) was previously revealed as a major determinant of steatosis in nonalcoholic fatty liver disease, ${ }^{20-22}$ and associated with advanced disease course in alcoholic liver disease. ${ }^{23-25}$ However, in CHC, there is still no definitive consensus. This may be explained by differences in sample size, ethnicity, HCV genotype, or HWE. ${ }^{26-28}$ In the present meta-analysis, the pooled results of all five studies showed that the genotype GG of PNPLA3 rs738409 (C>G) was associated with a higher risk of both advanced liver fibrosis and liver steatosis compared with CC or CG in CHC patients. Subgroup analysis found that the association with advanced liver fibrosis was stronger among Caucasian populations than Asian populations, although there were no statistically significant differences among them regarding age, BMI, or gender ratio. This result may be related to the interleukin-28B (IL28B) gene polymorphism, which has been confirmed as another factor associated with the severity of liver disease in $\mathrm{CHC}^{29}$

A previous study observed that IL28B-CC protected PNPLA3GG patients from steatosis, and in several studies the Asian population had a higher frequency of the favorable IL28B-CC genotype than Caucasians. ${ }^{30-32}$ Perhaps the same is true for fibrosis liver disease. More related research is needed to reveal the detailed mechanism.

We also stratified the subjects according to HCV genotype. The genotype GG of PNPLA3 rs738409 (C>G) increased the risk of advanced liver fibrosis and liver steatosis, no matter the infecting HCV genotype (HCV-1 and HCV-4 versus HCV-1, 2, 3 , and 4). Although the risk of advanced liver fibrosis and liver steatosis in $\mathrm{CHC}$ patients infected with HCV-1 or HCV-4 was higher than in those infected with HCV-2 or HCV-3 as well, the difference was not significantly different. This may be because the numbers in the second group were comparatively few. The patients of all these studies were native Caucasians, and in Caucasians, HCV-1 (especially HCV-1b) was found previously to be the most prevalent genotype. ${ }^{33}$ In each of the studies included in the present analysis, the proportion of patients infected with HCV-1 was more than $60 \%$. Thus, the proportion of patients infected with HCV-2 or HCV-3 was too small to influence this result. Studies with a greater number of patients infected with
HCV-2 or -3 are needed if we want to reveal more accurately the association between the PNPLA3 rs738409 (C>G) polymorphism and HCV genotype in liver disease progression of CHC.

This is the first meta-analysis concerning the relatedness of the PNPLA3 rs738409 (C>G) polymorphism and liver disease susceptibility in patients with CHC. Meta-analysis has become a new tool in genetic epidemiology and has been widely used, because it can enlarge the sample size and subsequently enhance the statistical power of quantitative analysis, to obtain more reliable conclusions. ${ }^{34}$ Before initiating this meta-analysis, we ensured that it was well designed with explicit methods for study selection, data extraction, and data analysis, and the quality of the included studies were all found sufficient (Table 2). No evidence of publication bias was detected, and the sensitivity analysis indicated that the results of the meta-analysis were stable.

This meta-analysis is limited in that only five studies were included, and therefore the sample size and statistical power may be insufficient. As more original studies are performed that investigate the association between PNPLA3 rs738409 (C>G) and liver disease susceptibility in patients with $\mathrm{CHC}$, data should be pooled to update this meta-analysis, especially for Asian populations or other ethnic groups. Furthermore, our lack of access to the original data of the studies limited further evaluations of the potential effects of the polymorphism on liver disease susceptibility. For example, from the articles there is no unified standard of diagnostic criteria or grading system for fibrosis and steatosis. Finally, although no publication bias was found by statistical analysis, this may have occurred since only published studies were included in the meta-analysis.

In conclusion, the results of this meta-analysis indicate that the genotype GG of PNPLA3 rs738409 (C>G) was associated with a higher risk of both advanced liver fibrosis and liver steatosis in patients with CHC, and especially among Caucasian compared with Asian cohorts. More research should be conducted to reveal how the PNPLA3 rs738409 (C>G) polymorphism and different ethnic, IL28B, or HCV genotypes are related in liver disease progression of CHC. This meta-analysis should be updated continually.

\section{CONFLICTS OF INTEREST}

No potential conflict of interest relevant to this article was 
reported.

\section{ACKNOWLEDGEMENTS}

This work was supported in part by grants from the National Natural Science Foundation of China (grant number: 81071338).

\section{REFERENCES}

1. Shepard CW, Finelli L, Alter MJ. Global epidemiology of hepatitis C virus infection. Lancet Infect Dis 2005;5:558-567.

2. Seeff LB. The history of the "natural history" of hepatitis C (19682009). Liver Int 2009;29 Suppl 1:89-99.

3. Niederau C, Lange S, Heintges T, et al. Prognosis of chronic hepatitis C: results of a large, prospective cohort study. Hepatology 1998;28:1687-1695.

4. Bochud PY, Cai T, Overbeck K, et al. Genotype 3 is associated with accelerated fibrosis progression in chronic hepatitis C. J Hepatol 2009;51:655-666.

5. Davis GL, Alter MJ, El-Serag H, Poynard T, Jennings LW. Aging of hepatitis C virus (HCV)-infected persons in the United States: a multiple cohort model of HCV prevalence and disease progression. Gastroenterology 2010;138:513-521.e6.

6. Leandro G, Mangia A, Hui J, et al. Relationship between steatosis, inflammation, and fibrosis in chronic hepatitis C: a meta-analysis of individual patient data. Gastroenterology 2006;130:1636-1642.

7. Missiha SB, Ostrowski M, Heathcote EJ. Disease progression in chronic hepatitis C: modifiable and nonmodifiable factors. Gastroenterology 2008;134:1699-1714.

8. Poynard T, Bedossa P, Opolon P. Natural history of liver fibrosis progression in patients with chronic hepatitis C: the OBSVIRC, METAVIR, CLINIVIR, and DOSVIRC groups. Lancet 1997;349:825832.

9. Wright M, Goldin R, Fabre A, et al. Measurement and determinants of the natural history of liver fibrosis in hepatitis $\mathrm{C}$ virus infection: a cross sectional and longitudinal study. Gut 2003;52:574579.

10. Stattermayer AF, Rutter K, Beinhardt S, et al. Association of the IL28B genotype with insulin resistance in patients with chronic hepatitis C. J Hepatol 2012;57:492-498.

11. Valenti L, Aghemo A, Stattermayer AF, et al. Implications of PNPLA3 polymorphism in chronic hepatitis $C$ patients receiving peginterferon plus ribavirin. Aliment Pharmacol Ther 2012;35: 1434-1442.

12. Stattermayer AF, Rutter K, Beinhardt S, et al. Role of FDFT1 polymorphism for fibrosis progression in patients with chronic hepatitis C. Liver Int 2014;34:388-395.

13. Miyashita M, Ito T, Sakaki M, et al. Genetic polymorphism in cyclooxygenase-2 promoter affects hepatic inflammation and fibrosis in patients with chronic hepatitis C. J Viral Hepat 2012;19:608614.

14. Trepo E, Pradat P, Potthoff A, et al. Impact of patatin-like phos- pholipase-3 (rs738409 C>G) polymorphism on fibrosis progression and steatosis in chronic hepatitis C. Hepatology 2011;54:60-69.

15. Stang A. Critical evaluation of the Newcastle-Ottawa scale for the assessment of the quality of nonrandomized studies in metaanalyses. Eur J Epidemiol 2010;25:603-605.

16. Valenti L, Nobili V, Al-Serri A, et al. The APOC3 T-455C and C$482 \mathrm{~T}$ promoter region polymorphisms are not associated with the severity of liver damage independently of PNPLA3 I148M genotype in patients with nonalcoholic fatty liver. J Hepatol 2011;55:1409-1414.

17. Jackson D, White IR, Riley RD. Quantifying the impact of between-study heterogeneity in multivariate meta-analyses. Stat Med 2012;31:3805-3820.

18. Peters JL, Sutton AJ, Jones DR, Abrams KR, Rushton L. Comparison of two methods to detect publication bias in meta-analysis. JAMA 2006;295:676-680.

19. Zintzaras E, Ioannidis JP. HEGESMA: genome search meta-analysis and heterogeneity testing. Bioinformatics 2005;21:3672-3673.

20. Romeo S, Kozlitina J, Xing C, et al. Genetic variation in PNPLA3 confers susceptibility to nonalcoholic fatty liver disease. Nat Genet 2008;40:1461-1465.

21. Rotman Y, Koh C, Zmuda JM, Kleiner DE, Liang TJ, Nash CR. The association of genetic variability in patatin-like phospholipase domain-containing protein 3 (PNPLA3) with histological severity of nonalcoholic fatty liver disease. Hepatology 2010;52:894-903.

22. Valenti L, Al-Serri A, Daly AK, et al. Homozygosity for the patatin-like phospholipase-3/adiponutrin I148M polymorphism influences liver fibrosis in patients with nonalcoholic fatty liver disease. Hepatology 2010;51:1209-1217.

23. Stickel F, Buch S, Lau K, et al. Genetic variation in the PNPLA3 gene is associated with alcoholic liver injury in Caucasians. Hepatology 2011;53:86-95.

24. Tian C, Stokowski RP, Kershenobich D, Ballinger DG, Hinds DA. Variant in PNPLA3 is associated with alcoholic liver disease. Nat Genet 2010;42:21-23.

25. Trepo E, Gustot T, Degre D, et al. Common polymorphism in the PNPLA3/adiponutrin gene confers higher risk of cirrhosis and liver damage in alcoholic liver disease. J Hepatol 2011;55:906-912.

26. Chung KC, Burns PB, Kim HM. A practical guide to meta-analysis. J Hand Surg Am 2006;31:1671-1678.

27. Dennis J, Hawken S, Krewski D, et al. Bias in the case-only design applied to studies of gene-environment and gene-gene interaction: a systematic review and meta-analysis. Int J Epidemiol 2011;40: 1329-1341.

28. Rosenthal R, DiMatteo MR. Meta-analysis: recent developments in quantitative methods for literature reviews. Annu Rev Psychol 2001;52:59-82.

29. Agundez JA, Garcia-Martin E, Maestro ML, et al. Relation of IL28B gene polymorphism with biochemical and histological features in hepatitis C virus-induced liver disease. PLoS One 2012;7:e37998.

30. Valenti L, Aghemo A, Stattermayer AF. Interaction between IL28B and PNPLA3 genotypes in the pathogenesis of steatosis in chronic 
hepatitis C non genotype-3 patients. J Hepatol 2012;56:1209-1210.

31. Balagopal A, Thomas DL, Thio CL. IL28B and the control of hepatitis C virus infection. Gastroenterology 2010;139:1865-1876.

32. Thomas DL, Thio CL, Martin MP, et al. Genetic variation in IL28B and spontaneous clearance of hepatitis C virus. Nature 2009;461: 798-801.

33. Smith DB, Bukh J, Kuiken C, et al. Expanded classification of hep- atitis C virus into 7 genotypes and 67 subtypes: updated criteria and genotype assignment web resource. Hepatology 2014;59:318327.

34. Thakkinstian A, McElduff P, D'Este C, Duffy D, Attia J. A method for meta-analysis of molecular association studies. Stat Med 2005; 24:1291-1306. 